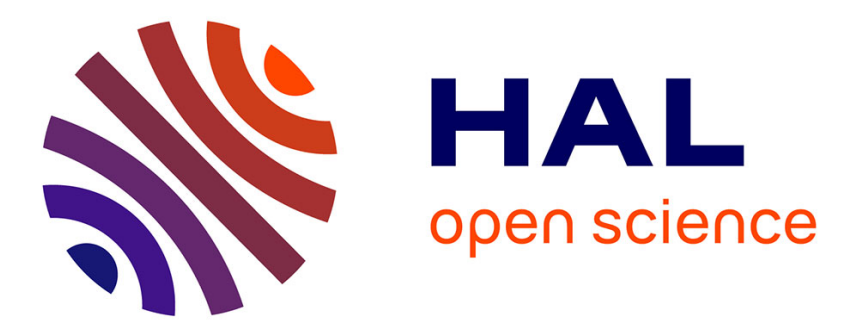

\title{
Pacifisme et violence: femmes et enfants dans la pédagogie de la paix d'Ernst Friedrich
}

\author{
Bérénice Zunino
}

\section{To cite this version:}

Bérénice Zunino. Pacifisme et violence: femmes et enfants dans la pédagogie de la paix d'Ernst Friedrich. Les Cahiers Sirice / Irice , 2011, 8, pp.111-136. 10.3917/lci.008.0111 . hal-03466879

\section{HAL Id: hal-03466879 https://hal.science/hal-03466879}

Submitted on 2 Jan 2022

HAL is a multi-disciplinary open access archive for the deposit and dissemination of scientific research documents, whether they are published or not. The documents may come from teaching and research institutions in France or abroad, or from public or private research centers.
L'archive ouverte pluridisciplinaire HAL, est destinée au dépôt et à la diffusion de documents scientifiques de niveau recherche, publiés ou non, émanant des établissements d'enseignement et de recherche français ou étrangers, des laboratoires publics ou privés. 


\title{
PACIFISME ET VIOLENCE : FEMMES ET ENFANTS DANS LA PÉDAGOGIE DE LA PAIX D'ERNST FRIEDRICH
}

\author{
B. Zunino, doctorante à l'Université Paris IV Sorbonne
}

Objecteur de conscience à vingt ans lorsque la Première Guerre mondiale éclate, Ernst Friedrich conçoit après-guerre une pédagogie de la paix qui se caractérise par une violence verbale et visuelle inouïe dont l'expression la plus cinglante est le recueil de photographies Guerre à la guerre! dévoilant des clichés effroyables des «Gueules cassées » et des images des conséquences tragiques du conflit sur l'environnement humain, naturel et matériel. ${ }^{1}$ Les enfants, soldats en puissance mais aussi incarnation de l'innocence, ${ }^{2}$ constituent une cible privilégiée de sa pédagogie. De fait, "l'enfance et l'adolescence apparaissent comme les moments privilégiés de la formation d'une culture $»^{3}$ Les femmes, qui furent peu sollicitées par les pacifistes, ${ }^{4}$ occupent également une place centrale dans son entreprise.

Dans une perspective d'histoire culturelle, les rôles des femmes et des enfants dans la pédagogie de la paix d'Ernst Friedrich nous confrontent à un double paradoxe. Par la place nouvelle qu'ils occupèrent dans ce conflit à caractère total, ${ }^{5}$ femmes et enfants représentèrent inévitablement un enjeu pour l'initiative de Friedrich, tant comme destinataires à convaincre que comme victimes dont il fallait montrer les souffrances. Ces deux catégories semblent pour Friedrich peser dans la construction de la paix, et a contrario dans le déroulement de la guerre. Or dans les représentations collectives, malgré les changements engendrés par la Première Guerre mondiale, femmes et enfants ne jouent pas de rôle dans la guerre, si ce n'est celui de victimes passives. ${ }^{6}$ Celle-ci reste un monde d'hommes et d'adultes. ${ }^{7}$ La brutalité de la pédagogie de Friedrich, qui a pourtant pour but de promouvoir un idéal de non-violence, est d'autant plus surprenante qu'elle s'adresse aux femmes et aux enfants, " emblèmes de la souffrance innocente ${ }^{8}$. Dans quelle mesure cette «violence pacifiste » qui «brutalise » des femmes et des enfants est-elle conciliable avec un idéal de paix ?

Il ne s'agit pas d'entamer une réflexion sur la dimension théorique de la conception de la guerre de Friedrich, ni de proposer une analyse de l'ensemble des destinataires et des fonctions de sa pédagogie de la paix. Ce qui nous intéressera, c'est la méthode brutale employée par Friedrich pour s'adresser aux femmes et aux enfants ainsi que les rôles spécifiques qu'il leur attribue au moment où il pose les fondements de sa pédagogie, entre la fin du conflit et la fondation de son Musée Antiguerre en 1925, qui couronne son entreprise pédagogique. Ces deux catégories peuvent parfois être envisagées de manière autonome, mais

\footnotetext{
${ }^{1}$ Ernst Friedrich, Krieg dem Kriege!, Munich, Deutsche Verlags-Anstalt, 2004. Première édition : Berlin, Freie Jugend Internationales Haus, 1924.

2 Stéphane Audoin-Rouzeau, La Guerre des enfants, 1914-1918, Paris, Colin, 2004. Historial de la Grande Guerre (éd.), Les enfants dans la Grande Guerre, Milan, Cinq Continents, 2003.

${ }^{3}$ Nicolas Offenstadt, «Contre la guerre. Ce que nous pouvons faire dans nos classes. L'École émancipée et la lutte pour la paix dans l'entre-deux-guerres », dans Institut d'histoire de la Révolution Française, Centre de recherche Espace, sociétés, cultures, Institut universitaire de formation des maîtres (éd.), Maurice Dommanget (1888-1976), citoyen, pédagogue, historien, Beauvais, Archives départementales de l'Oise, 1996, p. 79-87, ici p. 83. Marc Cluet (dir.), Le culte de la jeunesse et de l'enfance en Allemagne, 1870-1933, Rennes, PUR, 2003.

${ }_{5}^{4}$ Karl Holl, Pazifismus in Deutschland, Francfort/Main, Suhrkamp, 1988.

5 Gerd Krumeich, «Kriegsfront - Heimatfront », dans Gerhard Hirschfeld, Gerd Krumeich, Dieter Langewiesche, Hans-Peter Ullmann (dir.), Kriegserfahrungen, Studien zur Sozial- und Mentalitätsgeschichte des Ersten Weltkriegs, Essen, Klartext, 1997, p. 12-19. Anne Roerkohl, « Schlachtfeld Heimat », dans Christine Beil (dir.), Der Erste Weltkrieg, Hambourg, Rowohlt, 2004, p. 165-203.

${ }^{6}$ Laurent Gervereau, Montrer la guerre? Information ou propagande, Paris, Isthme, 2006.

${ }^{7}$ Claire Aslangul, Représentations de la guerre chez les peintres, graveurs et dessinateurs allemands au XXe siècle, dans le contexte européen : traditions, évolutions et ruptures dans les codes iconographiques, Jacques Le Rider, Gerd Schwerhoff, Ecole pratique des hautes études, 2003.

${ }^{8}$ Ibid., p. 96.
} 
par les interactions qu'elles entretiennent, certaines de leurs fonctions sont complémentaires. D'une part, Friedrich met l'accent sur le lien filial qui les unit et s'intéresse à l'influence des femmes sur les enfants, notamment à travers l'éducation, devoir traditionnellement réservé aux hommes. D'autre part, il soulève l'existence d'une autre forme d'influence moins évidente, celle de la reproduction du monde des adultes par les enfants, et plus particulièrement de la répartition traditionnelle des tâches. Autrement dit, il s'agit aussi de s'interroger dans une perspective genrée sur les liens entre féminité, violence et pacifisme.

Ces réflexions sur la violence féminine et sur le rôle des femmes dans le combat pour la paix correspondent à un sujet historiographique actuel. ${ }^{9}$ Elles amènent à analyser les stéréotypes féminins, dont le plus répandu est celui de la femme pacifique. ${ }^{10}$ En revanche, les recherches sur Friedrich sont peu nombreuses. ${ }^{11}$ Hormis une courte biographique, ${ }^{12}$ quelques articles traitent soit, d'un point de vue organisationnel, de son rôle d'orateur au sein du groupe de jeunesse «Freie Jugend » et auprès des enfants, ${ }^{13}$ soit de la violence des photos de son ouvrage Guerre à la guerre!, considéré comme le pilier d'une «culture de paix ». ${ }^{14}$ Pour appréhender notre sujet, nous étudierons des supports destinés aux femmes et aux enfants ou utilisant ces catégories comme agents mobilisateurs. Nous nous pencherons sur son livre de photographies, ainsi que sur des sources plus méconnues, comme le recueil de contes pour enfants Jardin d'enfants prolétarien, ${ }^{15}$ des articles du journal pour la jeunesse Jeunesse Libre et la brochure Le Musée Antiguerre. ${ }^{16}$

Dans une démarche synchronique, nous nous intéresserons aux fonctions des femmes et des enfants dans la pédagogie souvent violente de Friedrich et aux rapports des deux catégories à cette brutalité. Dans une attitude de rejet davantage antimilitariste que véritablement pacifiste, Friedrich cherche à «cultiver» le dégoût de la guerre chez les enfants, mais aussi chez les femmes, qui sont à la fois destinataires et intermédiaires de cette pédagogie violente considérée comme salvatrice. Érigées avant tout en victimes du conflit, femmes et enfants, dont on dévoile de manière mobilisatrice les souffrances endurées durant la guerre, permettent de déconstruire le mythe de la virilité qu'elles contribuent paradoxalement à alimenter. Par sa conception de la femme, et en particulier de la mère, douée d'un «instinct pacifiste», Friedrich parvient-il véritablement à proposer une redéfinition des genres, fondement d'une société pacifique?

\footnotetext{
${ }^{9}$ Cécile Dauphin, Arlette Farge (dir.), De la violence et des femmes, Paris, Albin Michel, 1997. Sabine Hering, Cornelia Wenzel, Frauen riefen aber man hörte sie nicht: die Rolle der deutschen Frauen in der internationalen Frauenfriedensbewegung zwischen 1892 und 1933, Cassel, Archiv der deutschen Frauenbewegung, 1986. Gisela Brinker-Gabler (dir.), Frauen gegen den Krieg, Francfort/Main, Fischer, 1980. Nous notons également l'organisation d'un séminaire sur le thème « pacifisme et violence » à l'ENS de Lyon.

${ }^{10}$ Cécile Dauphin, Arlette Farge, De la violence et des femmes, op. cit.

${ }^{11}$ Ernst Friedrich, préface de Gerd Krumeich, Krieg dem Kriege!, op. cit.

${ }^{12}$ Tommy Spree, Ich kenne keine „Feinde“. Der Pazifist Ernst Friedrich; ein Lebensbild, Berlin, Druck- und Verlagsgesellschaft mbH, Druckerei Conrad, 2000.

13 Heribert Baumann, «Libertäre Pädagogik in der Weimarer Republik, dargestellt am Beispiel der Kindergruppenarbeit von Ernst Friedrich und der Freien Jugend », dans Heribert Baumann (dir.), Werkstattbericht Pädagogik, Grafenau, Trotzdem, 1985, vol. 1, p. 63-88. Ulrich Linse, Die anarchistische und anarcho- syndikalistische Jugendbewegung 1918- 1933, Francfort/Main, dipa, 1976.

${ }^{14}$ Nicolas Offenstadt, «L'image contre la guerre. Autour d'Ernst Friedrich », dans Thérèse Blondet-Bisch (dir.), Voir, ne pas voir la guerre, Paris, Somogy, 2001, p. 271-275, ici p. 271. Sabine Kienitz, « Beschädigte Helden », dans Jost Dülffer, Gerd Krumeich (dir.), Der verlorene Frieden, Essen, Klartext, 2002, p. 199-214. Thomas Schirmböck, Roland Scotti, «Gibt es eine pazifistische Kriegsfotographie?», dans Dietrich Harth, Dietrich Schubert, Ronald Michael Schmidt (dir.), Pazifismus zwischen den Weltkriegen. Deutsche Schriftsteller und Künstler gegen den Krieg und Militarismus, Heidelberg, HVA, 1985, p. 218-228.

${ }^{15}$ Ernst Friedrich, Proletarischer Kindergarten. Ein Märchen- und Lesebuch für Gro $\beta$ und Klein, Berlin, Buchverlag der Arbeiter-Kunst-Ausstellung, 1921.

${ }^{16}$ Ernst Friedrich, Das Anti-Kriegsmuseum, Berlin, Anti-Kriegsmuseum, 1926.
} 


\section{«Guerre à la guerre ! : une éducation antimilitariste à la violence salvatrice}

«Apprenez aux enfants à refuser plus tard le service militaire !». ${ }^{17}$ L'appel de Friedrich à la responsabilité des parents dans le prologue de Guerre à la guerre! montre l'importance qu'il accorde à l'éducation pour la cause pacifiste. Les premières images de l'ouvrage sont ainsi consacrées à la dénonciation des dangers d'une éducation militariste, point de départ de son initiative. En guise d'introduction, on observe des reproductions de jouets de guerre tels que soldats de plomb, jeux de société à l'effigie d'Hindenburg ou encore canons en papier auxquels viennent s'ajouter manuels scolaires et livres d'images. Sur une couverture d'un des recueils reproduits, on reconnaît le conte des frères Grimm Le Roi Grenouille. Après cet étalage d'objets apparemment inoffensifs, le lecteur est invité à faire le lien entre cette éducation et ce qui est présenté comme sa conséquence, la guerre, illustrée dans les pages suivantes par des amoncellements de cadavres en putréfaction gisant sur les champs de bataille.

Ce procédé pédagogique de cause à effet se retrouve sur l'image Petites causes, grandes conséquences (Ill. 1), reproduite au début de l'ouvrage et diffusée à l'époque sous forme d'affiches et de tracts intitulés « Noël approche !». ${ }^{18}$ Celle-ci est consacrée à la dénonciation du jouet de guerre, support symptomatique de l'omniprésence quotidienne des valeurs militaires ${ }^{19}$ que Friedrich rejetait avec acharnement. A l'arrière-plan, un défilé de garçonnets coiffés de casques en papier brandissant des sabres en bois et des tambours fait écho au désespoir d'un homme venant de poignarder un de ses concitoyens. Dans ce tract qualifié de «manifeste de Noël $»,{ }^{20}$ les membres de la famille, et notamment les mères et les sœurs, sont exhortés à ne pas offrir de jouets de guerre aux enfants pour Noël, dont la symbolique chrétienne de solidarité, d'amour et de paix est rappelée. Ils seraient l'équivalent d'un «crime pédagogique », ${ }^{21}$ le «Juda » ${ }^{22}$ que les parents introduiraient innocemment dans le foyer et qui inculquerait dès le plus jeune âge une euphorie guerrière aux tout petits.

En cherchant à traquer cette mobilisation précoce, source de violence larvée, au sein de la sphère privée, Friedrich réagit aux phénomènes de banalisation et d'idéalisation de la guerre et, en creux, à l'autre face de la médaille, la brutalisation. ${ }^{23}$ Ce tract fait indéniablement écho à «l'enrôlement graphique des enfants dans le conflit ${ }^{24}$ aussi bien sur les photographies de famille - propre expérience de Friedrich, posant au côté de ses frères en uniforme de marin, expression de l'enthousiasme général pour la flotte de guerre allemande que sur de multiples supports de propagande, en particulier les cartes postales. Sur ces petits rectangles colorés, les enfants, projetés dans des responsabilités d'adultes tout en conservant leur candeur spécifique, à la fois mascottes de guerre attendrissantes et agents moralisateurs incarnant un idéal vers lequel les adultes doivent tendre, sont propices à mobiliser leurs

\footnotetext{
17 «Erzieht die Kinder so, daß sie sich später weigern, Soldaten- und Kriegsdienste zu tun! », Ernst Friedrich, Krieg dem Kriege!, op. cit., p. 9-10.

${ }^{18}$ Ernst Friedrich, «Weihnachten naht! », Freie Jugend, n 14, 1922. Kleine Ursachen, große Wirkungen, Ernst Friedrich, Krieg dem Kriege!, op. cit., p. 49.

${ }^{19}$ Heike Hoffmann, «'Schwarzer Peter im Weltkrieg': Die deutsche Spielwarenindustrie 1914-1918», dans Gerhard Hirschfeld, Gerd Krumeich, Dieter Langewiesche, Hans-Peter Ullmann, Kriegserfahrungen... op. cit., p. 323-335.

${ }_{20}$ «Weihnachtsmanifest », Freie Jugend, n $14,1922$.

${ }^{21}$ «Pädagogisches Verbrechen », Ernst Friedrich, Das Anti-Kriegsmuseum, op. cit., p. 11.

22 «Der Judas », Ernst Friedrich, Krieg dem Kriege!, op. cit., p. 10.

${ }^{23}$ George Mosse, De la Grande Guerre au totalitarisme : la brutalisation des sociétés européennes, Paris, Hachette-Littératures, 1999.

${ }^{24}$ François Pairault, Images de Poilus. La Grande Guerre en cartes postales, Paris, Tallandier, 2002, p. 89.
} 
parents ainsi qu'à renforcer les stéréotypes des genres. ${ }^{25}$ Friedrich avait certainement perçu le danger du fort pouvoir de minimisation des figures enfantines, auquel les femmes seraient particulièrement réceptives. $^{26}$

Dans cette perspective, Friedrich cherche à combattre l'aveuglement face à la banalisation de la guerre. Son entreprise est double : l'éducation pacifiste des enfants est inséparable d'une sensibilisation des parents, et en particulier des mères, à qui il confère, de manière révolutionnaire pour l'époque, un devoir d'éducation. ${ }^{27} \mathrm{Sa}$ réaction aux chansons militaires illustre l'importance de l'interdépendance entre femme et enfant dans sa pédagogie. Friedrich avertit que "la mère qui chante des chansons de soldats à l'enfant assis sur ses genoux prépare la guerre $»^{28}$ Face à cette ignorance et à ce complot militariste, il adopte une tactique de dévoilement ${ }^{29}$ dont un des ressorts consiste en l'explication crue et détaillée du vocabulaire militaire, premier seuil de la violence de sa pédagogie.

En réaction à une comptine militariste, La Chanson de l'épée, ${ }^{30}$ il s'attache à définir avec exactitude les fonctions de cette arme et ses effets sur la victime pour démystifier l'événement guerrier. Il dénonce à la fois la symbolique de cet attribut guerrier médiéval et chevaleresque particulièrement propice à minimiser la dimension destructrice d'une guerre moderne telle que la Première Guerre mondiale, et le danger propagandiste de la musique, moyen de persuasion très efficace. ${ }^{31}$ Il semble avoir d'autant mieux cerné la portée mobilisatrice de l'émotion qu'il la reprend à son compte en proposant une définition terrifiante :

[...] si à l'école on inculque aux enfants dès le plus jeune âge l'engouement pour le meurtre et les armes, qu'on explique alors avec un peu moins de 'poésie' et de rimes ce qu'est vraiment une 'épée'! En bon allemand: un long morceau d'acier finement affúté pourvu des deux côtés d'une pointe effilée et d'une longue rigole de sang. Et on a 'besoin' de cette épée pour transpercer les corps humains et déchirer les cerveaux humains! La longue rigole de sang sert à ce que, après avoir vite retiré 'l'épée', la blessure dans la poitrine de l'homme ainsi poignardé reste ouverte afin que le sang de l'être humain mourant puisse s'écouler lentement. ${ }^{32}$

Cette définition apparemment concrète et rationnelle vise en réalité à provoquer une réaction émotionnelle, qui serait l'équivalent négatif de celle engendrée dans la comptine. L'importance centrale de la cible dans cette description donne la désagréable impression au jeune lecteur d'être l'objet de l'attaque. Le dégoût et l'horreur se substituent à l'euphorie par un procédé d'identification suscitant l'empathie.

Conscient de la capacité humaine à l'abstraction, il élabore une pédagogie basée sur l'affect, non dépourvue d'une certaine brutalité, que l'on pourrait qualifier de pédagogie des sens. Friedrich joint à sa définition de l'épée la photographie d'une baïonnette afin d'illustrer, mais aussi, comme l'indique le verbe allemand de «veranschaulichen », de rendre sensible

\footnotetext{
${ }^{25}$ Bérénice Zunino, Les représentations des femmes et des enfants sur les cartes postales allemandes de la Première Guerre mondiale, Claire Aslangul, Université Paris IV Sorbonne, 2009. [Mémoire de Master 2]

${ }^{26}$ Ruth Diehl, Detlef Hoffmann, Ingrid Tabrizian (dir.), Ein Krieg wird ausgestellt, Francfort,/Main, Brönners Druckerei Breidenstein KG, 1976.

${ }^{27}$ Sabine Hering, Cornelia Wenzel, Frauen riefen aber man hörte sie nicht, op. cit.

28 « Die Mutter, die dem Kind auf ihrem Schoß, Soldatenlieder singt, bereitet Krieg vor! », Ernst Friedrich, Krieg dem Kriege!, op. cit., p. 10.

${ }^{29}$ Nicolas Offenstadt, «L'image contre la guerre. Autour d'Ernst Friedrich », art. cité.

30 «Das Schwertlied», Ernst Friedrich, Das Anti-Kriegsmuseum, op. cit., p. 6.

${ }^{31}$ Heinz Lemmermann, Kriegserziehung im Kaiserreich. Studien zur politischen Funktion von Schule und Schulmusik 1890-1918, Lilienthal/Brême, Eres Edition, 1984.

32 «...] wenn man die Kinder in der Schule schon für den Mord und die Mordwaffen begeistert, da $\beta$ man dann auch etwas weniger ,poetisch' und ungereimt aussprechen darf, was ein ,Schwert' überhaupt ist! Auf gut deutsch: ein langer haarscharf geschliffener Stahl mit einer feinen Spitze und einer langen Blutrinne auf beiden Seiten. Und ,gebraucht' wird dieses Schwert zum Stechen in Menschenleiber und zum Hauen in Menschengehirne! Die lange Blutrinne hat den Zweck: da $\beta$ nach dem schnellen Herausziehen des ,Schwertes' die Wunde in der Brust des also gestochenen Menschen geöffnet bleibt, damit das Blut des sterbenden Menschen langsam heraussickern kann. », Ernst Friedrich, Das Anti-Kriegsmuseum, op. cit., p. 6.
} 
les dangers de l'arme. Cette définition est d'autant plus persuasive qu'elle stimule plusieurs sens : l'image de la baïonnette, la description du sang et du corps transpercé de la victime, l'allitération en «f » («finement », «affûté », « effilée ») qui rappelle l'aspect tranchant de la lame, la longueur des phrases et les points d'exclamation, allusions au supplice causé par la blessure, mobilisent la vue, l'ouïe, le toucher et interpellent l'imagination. Cette brutalité apparaît chez Friedrich comme un moyen de persuasion constitutif de sa rhétorique passionnée dont il revendique la radicalité. Il confère à sa démarche une légitimité en opposant un combat mortel, incarné par la guerre moderne, à un combat pour la vie humaine, une « guerre des mots ${ }^{33}$ : sa pédagogie de l'horreur.

Cette notion de "guerre à la guerre » atteint son paroxysme dans l'ouvrage éponyme publié en 1924 dont la violence accrue des images témoignent des « vomissements de l'aprèsguerre $» .{ }^{34}$ Cette brutalité relatant les expériences du conflit n'est pas seulement verbale, elle est aussi visuelle ; «[...] la pédagogie pacifiste est une pédagogie du choc et de l'image. $»^{35}$ Les photographies des «Gueules cassées » et des cadavres, couplée aux commentaires de Friedrich, inspirent un dégoût presque physique et viscéral de la guerre moderne. L'objectif de l'ouvrage est bien de rappeler, au moment où les conséquences tragiques du conflit s'estompent, ${ }^{36}$ les expériences terribles de la Grande Guerre et de les ancrer dans les mémoires de celles et ceux que Friedrich qualifie, à la manière d'Henri Barbusse, de « machines à oublier ». ${ }^{37}$

Néanmoins, même si «[...] l'image pacifiste [...] passe très souvent par la représentation de la guerre et non de la paix », ${ }^{38}$ la dimension « destructrice » prépondérante de cette «pédagogie de la dissuasion ${ }^{39}$ ne confine-t-elle pas Friedrich dans une attitude de rejet qui le rapproche davantage de l'antimilitarisme que du pacifisme ? Lui-même se considère comme un « antimilitariste révolutionnaire » et ajoute que cet engagement est « tout autre chose que du pacifisme $\gg^{40}$ De fait, Friedrich ne se contente pas, contrairement à des pacifistes radicaux tels que Foerster, de dénoncer la militarisation de la société, ${ }^{41}$ mais voit dans l'Etat capitaliste la véritable cause structurelle de la guerre et de «l'empoisonnement» militariste des jeunes générations à travers les institutions et les supports qui leur sont adressés. ${ }^{42}$ Cette lecture anticapitaliste de la guerre, qui l'amène à prôner des moyens de lutte radicaux tels que le sabotage, accentue son rapport ambigu à la violence, qui trouve son expression la plus problématique dans la tautologie offensive «guerre à la guerre », reprise d'un slogan anarchiste. ${ }^{43}$

\footnotetext{
33 «Ein Krieg der Worte», Susanne Brandt, «Kriegssammlungen im Ersten Weltkrieg. Denkmäler oder Laboratoires d'histoire? », dans Gerhard Hirschfeld, Gerd Krumeich, Irina Renz (dir.), „, Keiner fühlt sich hier mehr als Mensch... “, Erlebnis und Wirkung des Ersten Weltkriegs, Essen, Klartext, 1993, p. 241-258, ici p. 246.

${ }^{34}$ Laurent Gervereau, L'Histoire du visuel au XXe siècle, Paris, Seuil, 2003, p. 138.

${ }^{35}$ Nicolas Offenstadt, «Contre la guerre. Ce que nous pouvons faire dans nos classes. L'École émancipée et la lutte pour la paix dans l'entre-deux-guerres », art. cité, p. 85.

${ }^{36}$ Annegret Jürgens-Kirchhoff, « Kunst gegen den Krieg im Antikriegsjahr 1924 », dans Jost Dülffer, Gerd Krumeich (dir.), Der verlorene Frieden, Essen, Klartext, 2002, p. 287-310.

37 «Vergeßmaschinen », Ernst Friedrich, Das Anti-Kriegsmuseum, op. cit., p. 4.

${ }^{38}$ Nicolas Offenstadt, «L'image contre la guerre. Autour d'Ernst Friedrich », art. cité, p. 271.

39 Edelgard Spaude-Schulze, Macht das Maul auf! Kinder- und Jugendliteratur gegen den Krieg in der Weimarer Republik, Würzburg, Königshausen \& Neumann, 1990. Bérénice Zunino, Ernst Friedrichs Friedenspädagogik mit Kindern und Jugendlichen zwischen 1919 und 1925, Claire Aslangul, Université Paris IV Sorbonne, 2008. [Mémoire de Master 1]

${ }^{40}$ Ernst Friedrich, «Menschen a.D., Betrachtungen über Menschen, Soldaten und Ungeziefer », Freie Jugend, $\mathrm{n}^{\circ} 7,1923$.

${ }^{41}$ Karl Holl, Pazifismus in Deutschland, op. cit.

${ }^{42}$ Ernst Friedrich, «Jugendvergiftung im Staat », Freie Jugend, n 4 , 1921. Ernst Friedrich, « Der Staat - eine Mörderschule! », Freie Jugend, n 7, 1919.

${ }^{43}$ Karl Holl, Helmut Donat (dir.), Die Friedensbewegung. Organisierter Pazifismus in Deutschland, Österreich und in der Schweiz, Düsseldorf, Econ Taschenbuch, 1983. Annegret Jürgens-Kirchhoff, « Kunst gegen den Krieg im Antikriegsjahr 1924 », art. cité.
} 
Davantage que sur le terme de «révolutionnaire », c'est sur celui d'« antimilitariste» que nous voulons nous attarder. Le Musée Antiguerre fondé en 1925 et dans lequel les photos terribles occupent une place centrale, symbolise de fait un «antimilitarisme des plus virulents $\gg .{ }^{44}$ C'est dans cet esprit que Friedrich conçoit sa pédagogie, comme l'indique le titre de l'écrit «Education à l'antimilitarisme », ${ }^{45}$ dans lequel il explique qu'il guide personnellement les enfants dans sa «galerie de l'horreur ${ }^{46}$ et leur demande de dessiner ce qu'ils en ont retenu. Le dessin intitulé Le meurtrier - Le soldat : la différence ? (Ill. 2) qu'il attribue à un des visiteurs, W. Jann, 13 ans, illustre cette attitude de rejet systématique des valeurs militaires. D'une part, le meurtrier, en civil, tête baissée, se tient avec recueillement devant l'homme qui gît à ses pieds. Tout porte à croire qu'il regrette son acte : la forêt, la nuit, le cinquième commandement insistent sur la transgression de l'interdit, qui renvoie le meurtrier à sa conscience, symbolisée par la chaîne. D'autre part, le soldat, reconnaissable à son uniforme, brandit ostensiblement sa baïonnette. La scène se déroule en plein jour et montre qu'il a accompli son forfait en toute impunité. La couronne de laurier au-dessus de sa tête, faisant écho au cinquième commandement, illustre la perversion absolue des valeurs morales en temps de guerre, ${ }^{47}$ dont la dénonciation se prolonge dans une critique des

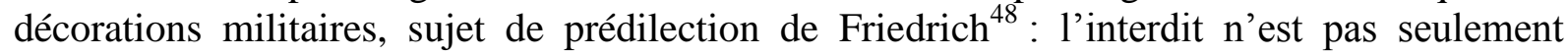
autorisé, il est aussi récompensé. Le cadavre sur lequel le militaire pose sa botte droite, est celui d'une femme, reconnaissable à sa robe. Il ne s'agit pas d'un affrontement guerrier «traditionnel », mais d'une scène d'atrocités, symbolisée par le sacrifice d'un civil et par la maison incendiée à l'arrière-plan. ${ }^{49}$ En guise de réponse à la question posée dans le titre du dessin, cette comparaison associée à l'exploitation de l'image de la femme comme victime innocente invite par conséquent à assimiler le soldat au meurtrier.

A cet égard, on peut se demander si le slogan «Les soldats sont des meurtriers », habituellement attribué à Kurt Tucholsky, n'est pas dû en réalité à Friedrich. De fait, ce dernier qualifie les soldats de «meurtriers professionnels ${ }^{50}$ et emploie la célèbre phrase antimilitariste dès 1921, dans son discours provocateur «Héros allemands » prononcé lors de l'inauguration d'un monument aux morts,${ }^{51}$ alors que la première occurrence n'apparaît chez Tucholsky que dix ans plus tard. ${ }^{52}$

Le dessin ne conduit pas seulement à assimiler meurtrier et soldat, il mène aussi à « sur-condamner » ce dernier, ce qui contribue paradoxalement, par la compassion qu'on peut ressentir à l'égard du meurtrier « repenti », à une sous-estimation de l'acte meurtrier. Or cette forme de banalisation de la violence ne rappelle-t-elle pas dans une moindre mesure l'augmentation du degré de tolérance envers les crimes politiques dans la République de Weimar ? La pédagogie de la paix de Friedrich, conçue en réaction à la brutalisation, n'en estelle pas paradoxalement le produit $?^{53}$ Dans un de ses articles antimilitaristes les plus virulents, «Etres humains a.D. Considérations sur les êtres humains, les soldats et la vermine $»,{ }^{54}$ Friedrich va jusqu'à assimiler les soldats à de la vermine en jouant sur le double sens du mot soldat en allemand (le militaire et l'insecte) et en exploitant les postulats de

\footnotetext{
${ }^{44}$ Ernst Friedrich, préface de Gerd Krumeich, Krieg dem Kriege!, op. cit., p. VIII.

${ }^{45}$ Ernst Friedrich, « Erziehung zum Antimilitarismus », dans Ernst Friedrich, Proletarischer Kindergarten, op. cit., p. 177-180.

46 «Galerie des Schreckens », Ernst Friedrich, Das Anti-Kriegsmuseum, op. cit., p. 25.

${ }^{47}$ Laurent Gervereau, Un siècle de manipulation par l'image, Paris, Somogy, 2000.

${ }^{48}$ Ernst Friedrich, « Deutsche Helden », Freie Jugend, n $29-30,1921$.

${ }^{49}$ Alan Kramer, «Les 'atrocités allemandes' : mythologie populaire, propagande et manipulations dans l'armée allemande », dans Jean-Jacques Becker, Jay Winter, Gerd Krumeich, Annette Becker, Stéphane Audoin-Rouzeau (dir.), Guerre et cultures (1914-1918), Paris, Colin, 1994, p. 147-164.

${ }^{50}$ «Berufsmörder », Ernst Friedrich, Das Anti-Kriegsmuseum, op. cit., p. 5.

${ }^{51}$ Ernst Friedrich, « Deutsche Helden », art. cité.

${ }^{52}$ Ignaz Wrobel, « Der bewachte Kriegsschauplatz », Die Weltbühne, n 31, 4.08.1931.

${ }^{53}$ Ernst Friedrich, préface de Gerd Krumeich, Krieg dem Kriege!, op. cit.

${ }^{54}$ Ernst Friedrich, « Menschen a.D. Betrachtungen über Menschen, Soldaten und Ungeziefer », art. cité.
} 
l'enfant innocent et de la femme pacifique. De fait, dans cet article provocateur où il relate une discussion avec sa fille de quatre ans qui se serait étonnée de voir durant une promenade un policier enlaçant une femme, il oppose le stéréotype de la femme pacifique et le penchant naturel de l'enfant pour la paix, qui trouve son expression dans la peur que ce dernier éprouve à la vue du soldat et qui serait progressivement pervertie par une éducation militariste, au manque d'humanité du soldat. Cette déshumanisation et cette «bestialisation » rappellent le traitement accordé à l'ennemi dans la propagande de 14-18 ainsi que le schéma de pensée manichéen caractéristique de l'époque. ${ }^{55}$

Néanmoins, la brutalisation qui caractérise Friedrich ne correspond pas seulement à un haut degré de violence mais est aussi le signe d'une nouvelle sensibilité. ${ }^{56}$ La violence qu'il emploie témoigne d'une réaction à fleur de peau face aux horreurs de la guerre moderne. En les racontant et en les montrant aux enfants, il cherche à atteindre ces derniers dans leur sentimentalité et à les dissuader de tout conflit. Sa stratégie de dévoilement, tout comme sa dénonciation de la militarisation du milieu scolaire et des jouets, rejoint la démarche de nombreux pacifistes de l'avant-1914 et de l'entre-deux-guerres, en particulier celle des instituteurs. ${ }^{57}$ Friedrich s'appuie notamment sur le roman de Wilhelm Lamszus publié en 1912, L'abattoir humain. Images de la prochaine guerre, dans lequel le potentiel destructeur des armes modernes est décrit de manière visionnaire. ${ }^{58}$

Il s'agit de contrecarrer l'influence perverse de l'éducation militariste de l'époque, autrement dit, non de faire naître, mais de développer, de «cultiver » à la manière des pédagogues rousseauistes un dégoût de la guerre latent chez les enfants, de nature pacifique et innocente. La brutalité et l'antimilitarisme de Friedrich sont par conséquent intrinsèquement liés aux problèmes structurels de la République de Weimar. Lors du choix de la dénomination de son musée, c'est par le caractère militariste de celle-ci qu'il justifie sa préférence pour la particule négative « anti ». ${ }^{59}$ Un « Musée de la Paix » ou «Musée Pacifiste » ${ }^{60}$ n'aurait pas eu sa place dans une société où la guerre est omniprésente. ${ }^{61}$ Son musée est conçu comme un véritable «sanatorium », ${ }^{62}$ sa «pédagogie du choc ${ }^{63}$ comme une thérapie. Employant la métaphore de la maladie pour désigner le virus de la guerre, Friedrich réinvente dans un esprit pacifiste et universaliste le slogan de l'Union Sacrée scandé par Guillaume II le 4 août 1914, «Je ne connais plus de partis, je ne connais désormais que des Allemands » : « Je ne connais pas d'ennemis. [...] Je ne connais que des êtres humains, que des êtres humains, en bonne santé ou malades. $»^{64}$

Si cette violence salvatrice consiste à entretenir chez les enfants un dégoût global de la guerre, le remède diffère néanmoins sensiblement selon le sexe des «patients ». Par l'attention particulière qu'il accorde à ces dernières, Friedrich se démarque de la plupart des

\footnotetext{
${ }^{55}$ George Mosse, De la Grande Guerre au totalitarisme : la brutalisation des sociétés européennes, op. cit.

56 Gerd Krumeich, «Die Präsenz des Kriegs im Frieden », dans Jost Dülffer, Gerd Krumeich (dir.), Der verlorene Frieden, Essen, Klartext, 2002, p. 7-17.

${ }^{57}$ Nicolas Offenstadt, «Contre la guerre. Ce que nous pouvons faire dans nos classes. L’École émancipée et la lutte pour la paix dans l'entre-deux-guerres », art. cité.

${ }^{58}$ Wilhelm Lamszus, Das Menschenschlachthaus. Bilder vom kommenden Krieg, Hamburg, Berlin, Janssen, 1912. Friedrich a été en contact avec cet instituteur et a publié des extraits de son roman. Voire : Freie Jugend, $\mathrm{n}^{\circ} 9,1919$.

59 «Anti-Kriegsmuseum », Ernst Friedrich, Vom Friedens-Museum zur Hitler-Kaserne. Ein Tatsachenbericht über das Wirken von Ernst Friedrich und Adolf Hitler, Books on Demand GmbH, Norderstedt, 2007, p. 25. Première édition : Gand, Schwarz, 1935.

${ }^{60}$ «Friedens-Museum », ibid., p. 25.

${ }^{61}$ Gerd Krumeich, « Die Präsenz des Kriegs im Frieden », art. cité.

${ }^{62}$ Ernst Friedrich, Das Anti-Kriegsmuseum, op. cit., p. 11.

${ }^{63}$ Nicolas Offenstadt, «L’image contre la guerre. Autour d'Ernst Friedrich », art. cité, p. 273.

64 «Ich kenne keine Feinde. [...] Ich kenne nur Menschen, nur Menschen, gesunde und kranke. » Ernst Friedrich, Vom Friedens-Museum zur Hitler-Kaserne..., op. cit., p. 53.
} 
initiatives pacifistes, qui privilégient la jeunesse masculine. ${ }^{65}$ Alors que cette aversion doit se substituer chez le petit garçon à l'admiration de l'uniforme, «blouse de meurtrier », ${ }^{66}$ elle doit remplacer chez les jeunes filles l'admiration pour le soldat. En réfléchissant au rôle du féminin dans le combat pour la paix, il remet en cause une certaine définition de la masculinité en construction lors de la Première Guerre mondiale : la virilité moderne. ${ }^{67}$

\section{La dénonciation du mythe de la virilité}

Une des fonctions spécifiques de sa pédagogie à l'adresse des femmes et des enfants consiste précisément à dénoncer ce mythe, qui contribue à la reproduction de stéréotypes genrés, dont l'admiration de la fillette pour le jeune soldat, représentée sur de nombreuses cartes postales, est un exemple. ${ }^{68}$ Friedrich effectue un lien, comme les féministes radicales et socialistes, ${ }^{69}$ entre militarisme et patriarcat.

Il propose aux garçonnets une analyse revue et corrigée du Petit Poucet de Ludwig Bechstein. ${ }^{70}$ Il remplace les attributs de force physique par des qualités mentales d'esprit critique et de générosité et érige Poucet en modèle de ruse et de solidarité, qu'il oppose à celui de l'homme viril. En faisant respectivement de l'ogre et de Poucet des personnifications du «Bourgeois » et du «Prolétaire », il livre un message anticapitaliste où virilité moderne est associée au capitalisme. Au même titre que ce dernier, les constructions du masculin, et $a$ contrario du féminin, constituent par conséquent pour Friedrich une des causes de la guerre, $a$ fortiori durant le conflit vu comme la guerre des sexes ${ }^{71}$ par excellence.

14-18 amorce de fait un changement dans la répartition des rôles en temps de guerre. Ce conflit total ou tendant à l'être fit des civils, dont les femmes et les enfants, de nouvelles cibles potentielles. ${ }^{72}$ Cette "porosité entre le front et l'arrière », ${ }^{73}$ qui s'accompagna d'une dissolution de «l'étanchéité entre les barrières de l'âge, qui exclut habituellement les enfants du monde combattant et de la violence de guerre ${ }^{74}$ explique l'importance de ces deux catégories dans la pédagogie de Friedrich. Le traitement brutal qui leur est accordé au sein de cette dernière reflète leurs expériences en temps de guerre. En réaction à une conception traditionnelle de la guerre véhiculée dans la propagande de l'époque qui mettait en scène un arrière épargné par les combats, ${ }^{75}$ il cherche à casser le mythe du cloisonnement entre le front

\footnotetext{
${ }^{65}$ Edelgard Spaude-Schulze, Macht das Maul auf! Kinder- und Jugendliteratur gegen den Krieg in der Weimarer Republik, op. cit.

${ }^{66}$ Ernst Friedrich, Krieg dem Kriege!, op. cit., p. 11.

${ }^{67}$ George Mosse, L'image de l'homme, l'invention de la virilité moderne, Paris, Abbeville, 1997.

${ }^{68}$ Katrin Kilian (dir.), Der Erste Weltkrieg in deutschen Bildpostkarten, vol. 66, Berlin, Directmedia Publishing, 2002. Bérénice Zunino, Les représentations des femmes et des enfants sur les cartes postales allemandes de la Première Guerre mondiale, op. cit.

${ }^{69}$ Sabine Hering, Cornelia Wenzel, Frauen riefen aber man hörte sie nicht, op. cit.

${ }^{70}$ Ludwig Bechstein, «Der kleine Däumling », dans Ernst Friedrich, Prolterischer Kindergarten, p. 8-12. Ernst Friedrich, « Helft einander! », dans ibid., p. 12-13.

${ }^{71}$ Françoise Thébaud, « Der Erste Weltkrieg, Triumph der Geschlechtertrennung », dans Georges Duby, Michèle Perrot, Heide Wunder, Geschichte der Frauen, vol. 5 : 20. Jahrhundert, Francfort, Campus, 1995, p. 33-91.

72 Anne Roerkohl, «Schlachtfeld Heimat», art. cité. Elke Koch, «,Jeder tut, was er kann fürs Vaterland": Frauen und Männer an der Heilbronner, Heimatfront' », dans Gerhard Hirschfeld, Gerd Krumeich, Dieter Langewiesche, Hans-Peter Ullmann, Kriegserfahrungen, Studien zur Sozial- und Mentalitätsgeschichte des Ersten Weltkriegs, p. 36-52.

${ }^{73}$ Ute Daniel, «Der Krieg der Frauen 1914-1918: Zur Innenansicht des Ersten Weltkriegs in Deutschland », dans Gerhard Hirschfeld, Gerd Krumeich, Irina Renz (dir.), „Keiner fühlt sich hier mehr als Mensch...", Erlebnis und Wirkung des Ersten Weltkriegs, Essen, Klartext, 1993, p. 131-149. Françoise Thébaud, «Femmes et genre dans la guerre », dans Stéphane Audoin-Rouzeau, Jean-Jacques Becker (dir.), Encyclopédie de la Grande Guerre 1914-1918, Histoire et Culture, Paris, Bayard, 2004, p. 613-625, ici p. 618.

${ }^{74}$ Manon Pignot, «Les enfants », dans ibid., p. 627-640, ici p. 627.

${ }^{75}$ Claire Aslangul, Représentations de la guerre chez les peintres, graveurs et dessinateurs allemands au XXe siècle..., op. cit.
} 
et l'arrière pour renvoyer à la réalité brutale. Dans Guerre à la Guerre !, les photos de villages détruits, brûlés ou bombardés, complétant les images des mutilés, symboles du bouleversement des genres et d'une ambiguïté entre les constructions de la féminité et de la masculinité, ${ }^{76}$ servent cette entreprise qui remet en cause l'image traditionnelle du guerrier. ${ }^{77}$

Dans cette optique, femmes et enfants «accèdent [...] au statut de symbole des horreurs de la guerre ${ }^{78}$ et deviennent des figures mobilisatrices. La photo intitulée «Des enfants arméniens morts de faim ${ }^{79}$ (Ill. 3) montrant des cadavres d'enfants morts lors du génocide rapproche ainsi les «blessures » de l'arrière à celles du front et montre que le corps des civils est également touché par la guerre. ${ }^{80}$ Face à une telle vision d'horreur, accentuée par la nudité du jeune corps au centre de l'image, le mythe d'une enfance globalement préservée des malheurs de la guerre véhiculé à l'époque par les cartes postales vole en éclat. ${ }^{81}$

Friedrich réfute aussi l'idée traditionnelle selon laquelle il serait impossible d'intégrer une femme dans un bataillon car cette dernière ne pourrait se définir par une dynamique violente. ${ }^{82}$ Le traitement de cette construction genrée trouve son expression complexe dans une photographie sur laquelle on peut voir le corps dénudé d'une femme qui gît sur le champ de bataille (Ill. 4). D'une part, Friedrich brise le tabou de la présence militaire de femmes au front durant la Grande Guerre, incompatible avec l'idéal suranné de la femme passive, qui fut, à contre-courant de la mobilisation intensive réelle des femmes durant le premier conflit mondial, paradoxalement véhiculé par les cartes postales de 14-18. ${ }^{83}$

D'autre part, le slogan couplé à l'image reprend le stéréotype de «la douceur des femmes dite constitutive $\gg{ }^{84}$ pour ériger la figure féminine en victime et dénoncer la guerre :

Un cadavre féminin violé ! (Il s'agit d'une femme issue d'un bataillon de la mort russe qui porte un uniforme d'homme. $)^{85}$

En distinguant des «formes de barbarie s'appliquant au masculin et au féminin ${ }^{86}$ l'exclamation indignée retire à la femme tout caractère violent et la conforte dans un rôle passif. Avant d'être actrice du conflit, la femme en est la victime, celle dont le corps devient un lieu de combat. ${ }^{87}$ La prise de vue, qui se focalise sur le sexe meurtri de la femme allongée et confère une position de domination aux trois soldats debout à l'arrière-plan, renforce cette

\footnotetext{
76 Sabine Kienitz, «Körper - Beschädigungen; Kriegsinvalidität und Männlichkeitskonstruktionen in der Weimarer Republik », dans Karen Hagemann, Stefanie Schüler-Springorum (dir.), Heimat-Front. Militär und Geschlechterverhältnisse im Zeitalter der Weltkriege, Francfort/Main, Campus, 2002, p. 188-207.

${ }^{77}$ Danièle Voldman, «Les bombardements aériens: une mise à mort du 'guerrier' ? (1914-1945) », dans Cécile Dauphin, Arlette Farge (dir.), De la violence et des femmes, Paris, Albin Michel, 1997, p. 146-158.

${ }^{78}$ Claire Aslangul, Représentations de la guerre chez les peintres, graveurs et dessinateurs allemands au XXe siècle..., op. cit., p. 100.

${ }^{79}$ Ernst Friedrich, Krieg dem Kriege!, op. cit., p. 154.

${ }^{80}$ Michael Geyer, «Violence et expérience de la violence au XXe siècle - la Première Guerre mondiale », dans Nicolas Beaupré, Anne Duménil, Christian Ingrao (dir.), 1914-1945, l'ère de la guerre. Violence, mobilisations, deuil, vol. 1 : 1914-1918, Paris, Viénot, 2004, p. 37-71.

${ }^{81}$ Bérénice Zunino, Les représentations des femmes et des enfants sur les cartes postales allemandes de la Première Guerre mondiale, op. cit.

${ }^{82}$ Karen Hagemann, «'Jede Kraft wird verbraucht'. Militäreinsatz von Frauen im Ersten und Zweiten Weltkrieg », dans Bruno Thoß, Hans-Erich Volkmann (dir.), Erster Weltkrieg, Zweiter Weltkrieg. Ein Vergleich. Krieg, Kriegserlebnis, Kriegserfahrung in Deutschland, Paderborn, Schöningh, 2002, p. 79-106. Yannick Ripa, «Armes d'hommes contre femmes désarmées: de la dimension sexuée de la violence dans la guerre civile espagnole », dans Cécile Dauphin, Arlette Farge (dir.), De la violence et des femmes, Paris, Albin Michel, 1997, p. 131-145.

${ }^{33}$ Bérénice Zunino, Les représentations des femmes et des enfants sur les cartes postales allemandes de la Première Guerre mondiale, op. cit.

${ }^{84}$ Yannick Ripa, «Armes d'hommes contre femmes désarmées : de la dimension sexuée de la violence dans la guerre civile espagnole », art. cité, p. 137.

85 «Geschändete Frauenleiche! (Es handelt sich um eine Frau aus dem russischen Todesbataillon, die männliche Soldatenkleider trägt.) », Ernst Friedrich, Krieg dem Kriege!, op. cit., p. 163.

${ }^{86}$ Cécile Dauphin, Arlette Farge, De la violence et des femmes, op. cit., p. 15.

${ }^{87}$ Stéphane Audoin-Rouzeau, L'enfant de l'ennemi, 1914-1918, Paris, Aubier, 1995.
} 
conception. L'explication objective qui complète l'exclamation émotive et vient contextualiser $^{88}$ la scène en précisant qu'il s'agit d'une femme russe, montre que Friedrich n'utilise pas l'image de la femme-victime pour susciter la haine inconditionnelle de l'ennemi, comme c'est le cas dans la propagande. ${ }^{89}$ Il ne démontre pas la brutalité inhérente à l'adversaire, mais celle qui est inhérente à la guerre. Ce n'est pas l'ennemi qui est accusé de transgresser les normes sociales, mais l'homme en guerre de manière générale. La condamnation du viol, considéré comme un moyen de domination et une expression de la virilité, ${ }^{90}$ va ici de pair avec le stéréotype d'une violence exclusivement masculine. Tout en dénonçant le mythe d'un front masculin, Friedrich conserve le stéréotype de la femme pacifique et conçoit surtout la guerre comme une «violence sur les femmes » et non comme une « violence des femmes ». ${ }^{91}$

En remettant en cause l'idée d'une complémentarité entre un arrière féminin et un front masculin, Friedrich s'attaque au mythe du soldat protecteur. ${ }^{92}$ Dans son discours « Héros allemands $",{ }^{93}$ il contredit cette représentation genrée selon laquelle le père de famille-soldat serait le garant de la sécurité des femmes et des enfants en insistant sur les souffrances de ces derniers durant le conflit. Il démontre ainsi l'absurdité de la guerre et la symbolique chauvine des monuments aux morts qui repose sur une dissimulation des souffrances. ${ }^{94}$

La tension entre femme-actrice du conflit et femme-victime se retrouve dans une photographie montrant un soldat coiffé d'un casque à pointe entouré de femmes aux seins nus (Ill. 5). Accompagnée d'un règlement promulgué par les autorités allemandes, cette image n'est pas conçue comme un chef d'accusation misogyne contre des femmes aux mœurs légères, mais présente la prostitution comme un phénomène symptomatique de la perte des valeurs morales inhérente à la guerre. C'est le mythe de l'épouse fidèle attendant patiemment le retour du guerrier au foyer, ainsi que le tabou des bordels militaires et l'hypocrisie de la morale sexuelle bourgeoise ${ }^{95}$ qui sont dénoncés. La femme y est à nouveau représentée en victime, d'autant plus que cette photographie figure dans l'ouvrage après celle de la femme violée. La dénonciation de la prostitution, assimilée à un viol, va de pair avec la condamnation de l'impérialisme allemand, comme le suggèrent ironiquement les célèbres vers chauvins accompagnant la photo, «et le monde sera régénéré par la nature allemande », ${ }^{96}$ dont la structure passive accentue l'image de la femme-victime. Le choix de Friedrich de faire figurer cette image sur la page de couverture de la première édition de Guerre à la guerre! illustre son caractère particulièrement scandaleux pour l'époque. ${ }^{97}$

Les femmes, avant tout présentées comme les victimes d'une « guerre masculine », ne sont pas seulement mobilisatrices pour la pédagogie de Friedrich. Le slogan d'une photographie montrant le cadavre abandonné d'un jeune soldat au bord d'une tranchée (Ill. 6) prouve qu'elles en sont aussi les destinataires : «Mères ! Voilà ce que fut le destin de vos fils

\footnotetext{
${ }^{88}$ Nicolas Offenstadt, «L'image contre la guerre. Autour d'Ernst Friedrich », art. cité.

${ }^{89}$ Yannick Ripa, «Armes d'hommes contre femmes désarmées : de la dimension sexuée de la violence dans la guerre civile espagnole », art. cité.

${ }^{90}$ Ibid. Stéphane Audoin-Rouzeau, L'enfant de l'ennemi, 1914-1918, op. cit.

${ }^{91}$ Cécile Dauphin, Arlette Farge, De la violence et des femmes, op. cit., p. 11.

${ }^{92}$ Birthe Kundrus, «Geschlechterkriege. Der Erste Weltkrieg und die Deutung der Geschlechterverhältnisse in der Weimarer Republik », dans Karen Hagemann, Stefanie Schüler-Springorum (dir.), Heimat-Front. Militär und Geschlechterverhältnisse im Zeitalter der Weltkriege, Francfort/Main, Campus, 2002, p. 171-187. George Mosse, L'image de l'homme, l'invention de la virilité moderne, op. cit.

${ }^{93}$ Ernst Friedrich, « Deutsche Helden », art. cité.

${ }^{94}$ George Mosse, De la Grande Guerre au totalitarisme : la brutalisation des sociétés européennes, op. cit.

${ }^{95}$ Siegfried Kogelfranz, «Schlange vorm Bordell », dans Stephan Burgdorff, Klaus Wiegrefe (dir.), Der Erste Weltkrieg. Die Urkatastrophe des 20. Jahrhunderts, Munich, DVA, 2004, p. 150-153.

96 « und es wird am deutschen Wesen einmal noch die Welt genesen », Ernst Friedrich, Krieg dem Kriege!, op. cit., p. 164.

${ }^{97}$ Ernst Friedrich, Krieg dem Kriege!, Berlin, Freie Jugend Internationales Haus, 1924.
} 
durant la guerre ! ${ }^{98}$ Friedrich s'adresse en particulier aux mères et reprend l'image consensuelle et mobilisatrice de la mater dolorosa. En ajoutant une description lapidaire du soldat, qui n'est plus anonyme puisqu'il prend les traits d'un fils, il attise délibérément la douleur de ces femmes qu'il touche au plus profond de leur chair et cherche ainsi à créer un sursaut chez celles qui «produisent» les soldats. On retrouve par conséquent une tension entre d'une part une femme-victime dont les souffrances culminent dans le deuil et d'autre part une femme en partie responsable du conflit puisqu'elle n'a pas su l'éviter. La portée universelle de cette interpellation est renforcée par la traduction en quatre langues (allemand, français, néerlandais, anglais), parfois maladroite, du texte qui accompagne l'image. Ce dernier fait écho à l'appel internationaliste que lance Friedrich dans le prologue : «Mères de tous les pays, unissez-vous ! ». ${ }^{99}$

En s'appuyant sur le bouleversement de la répartition traditionnelle des rôles masculins et féminins en guerre et en dénonçant violemment le mythe de la virilité, Friedrich tente de redéfinir les rapports homme-femme. Il attribue aux femmes, et en particulier aux mères, un rôle central dans la construction d'une société pacifique.

\section{« Mères de tous les pays, unissez-vous ! » : un « pacifisme féminin »?}

L'appel de Friedrich marque une véritable prise de conscience de l'importance des femmes pour sa pédagogie de la paix. Avec une attention particulière accordée aux mentalités, il cherche à provoquer un changement des comportements féminins, qu'il considère comme une des causes de la «violence masculine». Afin de mettre fin au mythe de la complémentarité entre le front et l'arrière, Friedrich ne se contente pas de dénoncer la fonction protectrice du guerrier, mais va jusqu'à inverser cette logique : c'est la femme qui n'a pas protégé l'homme, qui l'a laissé partir au front et a permis son sacrifice. En mettant en avant la fonction stabilisatrice des femmes en temps de guerre, il reconnait à ces dernières une part de responsabilité dans celle-ci. Si elles acceptent leur rôle passif et consentent en silence à la mobilisation des hommes, elles contribuent indirectement au maintien d'une société patriarcale et militariste. ${ }^{100}$

Cet appel repose sur une image universelle de la femme et sur une prédominance du sentiment. La figure féminine ne doit plus être celle à qui incombent le soin des blessés, le repos du guerrier et les larmes du deuil. Friedrich reprend l'idée d'un investissement sentimental des femmes en guerre, qui culmine dans la perte de l'être aimé, ${ }^{101}$ et les invite au contraire à montrer que «le lien de l'amour pour [leur] mari est plus fort que l'ordre de l'armée ! ${ }^{102}$ Il les appelle à renoncer à leur rôle d'épouses soumises et à empêcher par tous les moyens, quitte à «arracher les rails » ou à «se poster devant la locomotive », ${ }^{103}$ les hommes de partir au front s'ils ne trouvent pas eux-mêmes la force de déserter. Elles jouent par conséquent un rôle crucial pour la cause pacifiste dans la mesure où elles sont appelées à pallier la faiblesse masculine. Elles incarnent aux yeux de Friedrich le dernier espoir d'empêcher la guerre. Ce n'est pas un hasard s'il les interpelle en conclusion de son prologue de Guerre à la Guerre !. Cet appel aux épouses, basé sur la force du sentiment amoureux, culmine dans un appel aux mères, dont le potentiel pacifique est décuplé par la puissance de l'amour maternel, lien à la fois affectif et physique.

\footnotetext{
98 «Mütter! Das war das Schicksal eurer Söhne im Krieg [...]», Ernst Friedrich, Krieg dem Kriege!, op. cit., p. 93.

99 « Mütter aller Länder, vereinigt euch !», Ernst Friedrich, Krieg dem Kriege!, op. cit., p.12.

${ }^{100}$ Sabine Hering, Cornelia Wenzel, Frauen riefen aber man hörte sie nicht, op. cit.

${ }^{101}$ Stéphane Audoin-Rouzeau, Cinq deuils de guerre 1914-1918, Paris, Noesis, 2001.

102 «Zeigt, daß das Band der Liebe zu dem Gatten stärker ist, als der Armeebefehl! », Ernst Friedrich, Krieg dem Kriege!, op. cit., p.12.

${ }^{103}$ «Reißt alle Schienen auf, stellt Euch vor die Lokomotive! », Ibid., p. 12.
} 
Dans une société où maternité et éducation sont perverties en devoir patriotique, Friedrich exhorte les mères à jouer un rôle actif dans l'éducation des enfants et à poser ainsi les fondements d'une société pacifique. Dans cette perspective, on comprend d'autant mieux l'importance de sensibiliser les femmes à la cause pacifiste. L'image intitulée D'abord le jeu, ensuite l'enfer ${ }^{104}$ (Ill. 7), d'origine néerlandaise, met en scène une mère douce et aimante qui retire avec bienveillance des jouets de guerre des mains de son petit garçon. Le titre, dont la structure binaire rappelle celui de l'image Causes minimes, conséquences graves reproduite sur la même double-page, fait le lien entre ces jouets militaristes et le consentement à la guerre. Le personnage féminin, qui a tiré les enseignements de cet avertissement, incarne un modèle de comportement responsable.

Le rôle d'éducatrices que Friedrich attribue aux femmes dans cette représentation témoigne d'un progressisme qui tranche avec leur statut dans la société de l'époque. Selon le Code Civil, le droit à l'éducation des enfants incombait exclusivement aux pères de famille. La conception assez « révolutionnaire » de Friedrich rejoint de fait les revendications de nombreuses féministes. Lors du Congrès international des femmes à La Haye en 1915, le droit des femmes à l'éducation des enfants constitue l'une des revendications et l'un des moyens pour les «femmes et les mères d'Allemagne ${ }^{105}$ de se mobiliser contre la guerre. Lors de la Conférence de la Ligue internationale des femmes pour la paix et la liberté à Zürich en 1919, les militantes prônent la mise en place d'un Conseil d'éducation international permanent afin d'assurer une éducation dans l'esprit de la réconciliation des peuples et condamnent fermement la militarisation des supports pour enfants et notamment les jouets de guerre. ${ }^{106}$ Cette conception de la femme comme éducatrice est également partagée par les socialistes féministes ${ }^{107}$ et trouve des échos dans des initiatives individuelles : la féministe pacifiste Selma Lagerlöf considère que l'éducation des enfants dans le souvenir des horreurs de la guerre relève de la responsabilité des femmes. ${ }^{108}$ Ses propositions ainsi que la spécificité féminine qu'elle défend se rapprochent de la pédagogie de l'horreur et de la dissuasion de Friedrich.

Tout en complétant de manière constructive le versant antimilitariste de sa pédagogie de la paix, ce «pacifisme féminin » est en réalité conçu, comme en témoigne l'image précitée, comme un «devoir de maternité » ${ }^{109}$ envers la société. Convaincu de l'existence de prédispositions féminines pacifiques, Friedrich oppose à sa définition agressive du soldat le stéréotype de la femme pacifique. «Equation[s] paix/féminité » et «soldat/virilité » ${ }^{110}$ vont ici de pair :

«Si j'étais un peintre et que je devais faire un tableau représentant la vie et la mort, je peindrais

une femme enceinte main dans la main avec un soldat. ${ }^{I 1}$

Dans cette métaphore, la femme, symbole de fécondité et de vie par opposition au soldat, incarnation de la mort, est présentée comme la figure à la fois antithétique et complémentaire de l'homme brutal à travers laquelle Friedrich entrevoit un échappatoire à la violence. A ses yeux, cette fonction maternelle confère à la femme un caractère pacifique, qui ne détermine pas seulement le rôle qu'elle doit jouer dans la société, mais correspond aussi à une de ses

\footnotetext{
${ }^{104}$ Erst das Spiel, dann die Hölle, Ernst Friedrich, Krieg dem Kriege!, op. cit., p. 48.

${ }^{105}$ Gisela Brinker-Gabler, Frauen gegen den Krieg, op. cit., p. 180.

${ }^{106}$ Ibid.

${ }^{107}$ Sabine Hering, Cornelia Wenzel, Frauen riefen aber man hörte sie nicht, op. cit.

108 Selma Lagerlöf, « Auf uns kommt es jetzt an », dans Gisela Brinker-Gabler (dir.), Frauen gegen den Krieg, Francfort/Main, Fischer, 1980, p. 239-241.

109 «Mütterlichkeitsauftrag », Sabine Hering, Cornelia Wenzel, Frauen riefen aber man hörte sie nicht, op. cit., p. 94.

${ }^{110}$ Danièle Voldman, «Les bombardements aériens: une mise à mort du 'guerrier' ? (1914-1945) », art. cité, p. 147.

111 «Wenn ich ein Maler wäre und hätte die Aufgabe, ein Bild zu schaffen, das Leben und den Tod darstellend, ich würde ein schwangeres Weib malen Hand in Hand mit einem Soldaten. », Ernst Friedrich, « Menschen a.D. Betrachtungen über Menschen, Soldaten und Ungeziefer », art. cité.
} 
qualités intrinsèques de laquelle découle un «catalogue de vertus » ${ }^{112}$ telles que le dévouement, la douceur et la bonté.

Autrement dit, l'instinct maternel va de pair selon Friedrich avec un «instinct pacifique ». Cette conception essentialiste rejoint l'argumentation biologique de certaines féministes pacifistes telles que Lida Gustava Heymann et Olive Schreiner, qui opposent, avec certaines nuances, la violence et la dureté masculines à la clémence féminine. ${ }^{113}$ Dans cette perspective genrée qui vise à légitimer un «pacifisme féminin ", l'établissement d'une paix durable ne peut se concevoir que comme une victoire du «principe féminin » sur le «principe masculin $\gg{ }^{114}$ qui régit l'ensemble des sociétés et engendre la guerre. Comme le fait Friedrich, elles valorisent leurs fonctions maternelles spécifiques et leur propre investissement sentimental dans la guerre, qui culmine dans la souffrance du deuil, pour revendiquer un droit de regard sur tout conflit.

Or cette argumentation biologique qui exploite l'image de la femme permet-elle à Friedrich de surmonter la définition traditionnelle des genres dont il prétend s'affranchir ? En lui attribuant une «nature pacifique », il confine la femme dans une attitude de dévouement, de naïveté, voire de passivité. ${ }^{115}$ Contrairement à une lecture socialiste, Friedrich n'avertit pas en priorité du poids économique des femmes dans l'économie de guerre. ${ }^{116}$ Comme l'illustre la nouvelle de Berta Lask Comment le caporal Gottlieb Fürchtegott reçu l'ordre de prier, publiée dans le recueil Jardin d'enfants prolétarien, la femme dont le travail dans les usines à munitions dégrade la santé, est avant tout victime de la guerre, au même titre que l'enfant, incarnation de la pureté et de l'innocence. ${ }^{117}$ C'est en eux que le caporal trouve la sérénité divine qu'il cherchait en vain au sein des institutions cléricales militaristes.

Le message poétique et spirituel véhiculé par cette nouvelle reflète une vision apolitique de la femme. Alors que les féministes radicales et socialistes s'appuient sur cette argumentation essentialiste pour revendiquer des droits politiques, Friedrich ne rattache pas sa conception de la figure féminine à son analyse anticapitaliste. ${ }^{118}$ Contrairement à l'engagement pacifiste des féministes rassemblées à La Haye en 1915, qui proposèrent aussi des solutions d'ordre économique et abordèrent des questions de politique étrangère pour construire un ordre de paix, la contribution pacifique des femmes se limite chez Friedrich à un rôle socialisateur et moral, qui rappelle le conservatisme du mouvement féministe bourgeois. ${ }^{119}$ Cette dépolitisation, signe de sa pédagogie de l'affect, est au cœur de l'appel «Mères de tous les pays, unissez-vous ! », qui se base sur une définition privée et affective et non sur une conscience politique comme c'est le cas dans le Manifeste du Parti Communiste. Friedrich n'appelle pas véritablement à la fondation d'un «mouvement féminin pour la paix ${ }^{120}$ qui aurait une légitimité politique, ni à un sursaut organisé des féministes, dont beaucoup ont renoncé à leur engagement lors de la mise en place de l'Union Sacrée. ${ }^{121} \mathrm{La}$ femme reste à ses yeux l'ambassadrice universelle d'un idéal de paix. Convaincu de

\footnotetext{
112 «Tugendkatalog », Petra Müller, «Sind Frauen friedfertig? », Gegen welchen Krieg - für welchen Frieden? Beiträge zur feministischen Theorie und Praxis, ${ }^{\circ}$ 8, 1983, p. 27-32, ici p. 27.

${ }^{113}$ Lida Gustava Heymann, «Weiblicher Pazifismus », dans Gisela Brinker-Gabler (dir.), Frauen gegen den Krieg, Francfort/Main, Fischer, 1980, p. 65-70. Olive Schreiner, «Frauen zahlen die Hauptkosten », dans ibid., p. 45-50.

114 «Das männliche Prinzip », Lida Gustava Heymann, «Weiblicher Pazifismus », art. cité, p. 65.

${ }^{115}$ Petra Müller, « Sind Frauen friedfertig? », art. cité.

${ }^{116}$ Sabine Hering, Cornelia Wenzel, Frauen riefen aber man hörte sie nicht, op. cit.

${ }^{117}$ Berta Lask, «Wie der Gefreite Gottlieb Fürchtegott zum Beten kommandiert wurde », dans Ernst Friedrich, Proletarischer Kindergarten, op. cit., p. 68-72.

118 Sabine Hering, Cornelia Wenzel, Frauen riefen aber man hörte sie nicht, op. cit. Gisela Brinker-Gabler, Frauen gegen den Krieg, op. cit.

${ }^{119}$ Ibid.

120 «Frauenfriedensbewegung », Sabine Hering, Cornelia Wenzel, Frauen riefen aber man hörte sie nicht, op. cit., p. 3.

${ }^{121}$ Karl Holl, Pazifismus in Deutschland, op. cit. Gisela Brinker-Gabler, Frauen gegen den Krieg, op. cit.
} 
l'existence de prédispositions féminines pacifiques, il n'envisage pas non plus d'enthousiasme patriotique féminin, pourtant partagé par de nombreuses femmes durant le conflit. ${ }^{122}$ Il reste prisonnier du stéréotype de la femme pacifique et d'une idéalisation de la figure féminine qui retire à cette dernière toute crédibilité politique.

Or une telle conception de la femme - et par conséquent de la paix - a des répercussions sur la manière dont est envisagé le rapport du masculin au pacifisme. Les hommes ne deviendraient pacifiques qu'en se «féminisant», en intériorisant des valeurs proprement féminines. En souhaitant un «transfert ${ }^{123}$ - pacifique et unilatéral - du féminin vers le masculin, Friedrich ne parvient pas en réalité à s'affranchir du stéréotype des genres et de la logique patriarcale. ${ }^{124}$ Envisagée exclusivement comme un engagement des sœurs, des épouses et des mères, la contribution féminine à la cause pacifiste reste systématiquement définie par rapport au masculin. Dans le prolongement de cette logique, l'homme ne semble pas non plus être porté par une dynamique pacifique propre. Contrairement à d'autres pacifistes tels que Kurt Hiller, Friedrich ne considère pas qu'une aspiration autonome à la paix, motivée par leur refus de souffrir ou leur paternité, puisse émaner des hommes. ${ }^{125}$ Malgré son combat contre le mythe de la virilité, Friedrich continue à associer la masculinité à la guerre. C'est peut-être en partie dans une telle conception genrée de la paix que réside la faille de la pédagogie de Friedrich et plus généralement d'un pacifisme idéaliste pour lequel "l'aspiration à un état de paix n'apparaît jusqu'à aujourd'hui pas conciliable avec le mythe de la masculinité. » 126

La pédagogie de l'horreur de Friedrich se rapproche à maints égards d'une attitude de rejet et d'un antimilitarisme primaire dont la brutalité, fondement d'une pédagogie des sens et de l'affect, fonctionne comme un moyen de dissuasion et de persuasion. Cette radicalité s'explique également par des caractéristiques structurelles de l'entre-deux-guerres en Allemagne: en «brutalisant» femmes et enfants, Friedrich reproduit plus ou moins consciemment le schéma de pensée inhérent à son temps. Il dévoile également ainsi les véritables expériences de ces catégories durant cette guerre caractère total. Présentées avant tout comme les victimes innocentes du conflit, femmes et enfants ne sont pas seulement destinataires, mais aussi vecteurs de sa pédagogie de la paix à la violence mobilisatrice. Ils contribuent à remettre en question le mythe de la virilité et la représentation d'un arrière féminin et enfantin protégé des exactions guerrières par opposition à un front exclusivement masculin. Figures idéalisées à «l'instinct pacifiste » et agents socialisateurs, les femmes sont aux yeux de Friedrich doublement mobilisatrices pour la cause pacifiste. Cette perspective genrée reposant sur l'idée d'un " pacifisme féminin » permet à Friedrich de dépasser dans une certaine mesure la violence nihiliste de son antimilitarisme, qui constitue en réalité le point de départ de la construction d'une société pacifique. Mais sa conception du féminin, teintée de progressisme, n'aboutit pas véritablement à une redéfinition des genres. Chargée d'une mission éducatrice auprès des enfants, la femme reste avant tout déterminée par sa maternité et par une certaine passivité, qui trouve son expression dans l'image consensuelle de la mater dolorosa.

122 Brunhilde Sauer-Burghard, «'Nur wenige Frauen haben nicht mitgemacht', Feminismus gegen Militarismus? », Gegen welchen Krieg - für welchen Frieden? Beiträge zur feministischen Theorie und Praxis, $\mathrm{n}^{\circ} 8,1983$, p. 33-45.

${ }^{123}$ Danièle Voldman, «Les bombardements aériens: une mise à mort du 'guerrier' ? (1914-1945) », art. cité.

${ }^{124}$ Petra Müller, « Sind Frauen friedfertig? », art. cité.

${ }^{125}$ Sabine Hering, Cornelia Wenzel, Frauen riefen aber man hörte sie nicht, op. cit.

126 « [...] weil der Wunsch nach Friede mit dem Männlichkeitsmythos bis heute unvereinbar erscheint. », ibid., p. 95. 


\section{Bibliographie}

Claire Aslangul, Représentations de la guerre chez les peintres, graveurs et dessinateurs allemands au XXe siècle, dans le contexte européen : traditions, évolutions et ruptures dans les codes iconographiques, Jacques Le Rider, Gerd Schwerhoff, Ecole pratique des hautes études, 2003.

Stéphane Audoin-Rouzeau, Cinq deuils de guerre 1914-1918, Paris, Noesis, 2001.

Stéphane Audoin-Rouzeau, Jean-Jacques Becker (dir.), Encyclopédie de la Grande Guerre 1914-1918, Histoire et Culture, Paris, Bayard, 2004.

Stéphane Audoin-Rouzeau, La Guerre des enfants, 1914-1918, Paris, Colin, 2004.

Stéphane Audoin-Rouzeau, L'enfant de l'ennemi, 1914-1918, Paris, Aubier, 1995.

Heribert Baumann (dir.), Werkstattbericht Pädagogik, Grafenau, Trotzdem, 1985, vol. 1.

Heribert Baumann, «Libertäre Pädagogik in der Weimarer Republik, dargestellt am Beispiel der Kindergruppenarbeit von Ernst Friedrich und der Freien Jugend », dans Heribert Baumann, Werkstattbericht Pädagogik, op. cit., p. 63-88.

Nicolas Beaupré, Anne Duménil, Christian Ingrao (dir.), 1914-1945, l'ère de la guerre. Violence, mobilisations, deuil, vol. 1 : 1914-1918, Paris, Viénot, 2004.

Jean-Jacques Becker, Jay Winter, Gerd Krumeich, Annette Becker, Stéphane Audoin-Rouzeau (dir.), Guerre et cultures (1914-1918), Paris, Colin, 1994.

Christine Beil (dir.), Der Erste Weltkrieg, Hambourg, Rowohlt, 2004.

Thérèse Blondet-Bisch (dir.), Voir, ne pas voir la guerre, Paris, Somogy, 2001.

Susanne Brandt, «Kriegssammlungen im Ersten Weltkrieg. Denkmäler oder Laboratoires d'histoire? », dans Gerhard Hirschfeld, Gerd Krumeich, Irina Renz, ,,Keiner fühlt sich hier mehr als Mensch... “, Erlebnis und Wirkung des Ersten Weltkriegs, op. cit., p. 241-258.

Gisela Brinker-Gabler (dir.), Frauen gegen den Krieg, Francfort/Main, Fischer, 1980.

Stephan Burgdorff, Klaus Wiegrefe (dir.), Der Erste Weltkrieg. Die Urkatastrophe des 20. Jahrhunderts, Munich, DVA, 2004.

Marc Cluet (dir.), Le culte de la jeunesse et de l'enfance en Allemagne, 1870-1933, Rennes, PUR, 2003.

Ute Daniel, « Der Krieg der Frauen 1914-1918: Zur Innenansicht des Ersten Weltkriegs in Deutschland », dans

Gerhard Hirschfeld, Gerd Krumeich, Irina Renz, ,,Keiner fühlt sich hier mehr als Mensch... “, Erlebnis und Wirkung des Ersten Weltkriegs, op. cit., p. 131-149.

Cécile Dauphin, Arlette Farge (dir.), De la violence et des femmes, Paris, Albin Michel, 1997.

Ruth Diehl, Detlef Hoffmann, Ingrid Tabrizian (dir.), Ein Krieg wird ausgestellt, Francfort,/Main, Brönners Druckerei Breidenstein KG, 1976.

Georges Duby, Michèle Perrot, Heide Wunder, Geschichte der Frauen, vol. 5 : 20. Jahrhundert, Francfort, Campus, 1995.

Jost Dülffer, Gerd Krumeich (dir.), Der verlorene Frieden, Essen, Klartext, 2002.

Ernst Friedrich, Das Anti-Kriegsmuseum, Berlin, Anti-Kriegsmuseum, 1926.

Ernst Friedrich, « Der Staat - eine Mörderschule! », Freie Jugend, n 7, 1919.

Ernst Friedrich, « Deutsche Helden », Freie Jugend, n ${ }^{\circ} 29-30,1921$.

Ernst Friedrich, «Jugendvergiftung im Staat », Freie Jugend, n4, 1921.

Ernst Friedrich, Krieg dem Kriege!, Munich, Deutsche Verlags-Anstalt, 2004. Première édition : Berlin, Freie Jugend Internationales Haus, 1924.

Ernst Friedrich, « Menschen a.D., Betrachtungen über Menschen, Soldaten und Ungeziefer », Freie Jugend, $\mathrm{n}^{\circ} 7$, 1923.

Ernst Friedrich, Proletarischer Kindergarten. Ein Märchen- und Lesebuch für Gro $\beta$ und Klein, Berlin, Buchverlag der Arbeiter-Kunst-Ausstellung, 1921.

Ernst Friedrich, Vom Friedens-Museum zur Hitler-Kaserne. Ein Tatsachenbericht über das Wirken von Ernst Friedrich und Adolf Hitler, Berlin, Books on Demand GmbH, Norderstedt, 2007. Première édition : Gand, Schwarz, 1935

Ernst Friedrich, « Weihnachten naht! », Freie Jugend, n 14, 1922.

Laurent Gervereau, L'Histoire du visuel au XXe siècle, Paris, Seuil, 2003.

Laurent Gervereau, Montrer la guerre ? Information ou propagande, Paris, Isthme, 2006.

Laurent Gervereau, Un siècle de manipulation par l'image, Paris, Somogy, 2000.

Michael Geyer, "Violence et expérience de la violence au XXe siècle - la Première Guerre mondiale », dans Nicolas Beaupré, Anne Duménil, Christian Ingrao, 1914-1945, l'ère de la guerre. Violence, mobilisations, deuil, op. cit., p. 37-71.

Karen Hagemann, «'Jede Kraft wird verbraucht'. Militäreinsatz von Frauen im Ersten und Zweiten Weltkrieg », dans Bruno Thoß, Hans-Erich Volkmann, Erster Weltkrieg, Zweiter Weltkrieg..., op. cit., p. 79-106.

Karen Hagemann, Stefanie Schüler-Springorum (dir.), Heimat-Front. Militär und Geschlechterverhältnisse im Zeitalter der Weltkriege, Francfort/Main, Campus, 2002. 
Dietrich Harth, Dietrich Schubert, Ronald Michael Schmidt (dir.), Pazifismus zwischen den Weltkriegen. Deutsche Schriftsteller und Künstler gegen den Krieg und Militarismus, Heidelberg, HVA, 1985.

Sabine Hering, Cornelia Wenzel, Frauen riefen aber man hörte sie nicht: die Rolle der deutschen Frauen in der internationalen Frauenfriedensbewegung zwischen 1892 und 1933, Cassel, Archiv der deutschen Frauenbewegung, 1986.

Lida Gustava Heymann, «Weiblicher Pazifismus », dans Gisela Brinker-Gabler, Frauen gegen den Krieg, op. cit., p. 65-70.

Gerhard Hirschfeld, Gerd Krumeich, Dieter Langewiesche, Hans-Peter Ullmann (dir.), Kriegserfahrungen, Studien zur Sozial- und Mentalitätsgeschichte des Ersten Weltkriegs, Essen, Klartext, 1997.

Gerhard Hirschfeld, Gerd Krumeich, Irina Renz (dir.), ,, Keiner fühlt sich hier mehr als Mensch... “, Erlebnis und Wirkung des Ersten Weltkriegs, Essen, Klartext, 1993.

Historial de la Grande Guerre (dir.), Les enfants dans la Grande Guerre, Milan, Cinq Continents, 2003.

Heike Hoffmann, «'Schwarzer Peter im Weltkrieg': Die deutsche Spielwarenindustrie 1914-1918», dans Gerhard Hirschfeld, Gerd Krumeich, Dieter Langewiesche, Hans-Peter Ullmann, Kriegserfahrungen... op. cit., p. 323-335.

Karl Holl, Helmut Donat (dir.), Die Friedensbewegung. Organisierter Pazifismus in Deutschland, Österreich und in der Schweiz, Düsseldorf, Econ Taschenbuch, 1983.

Karl Holl, Pazifismus in Deutschland, Francfort/Main, Suhrkamp, 1988.

Institut d'histoire de la Révolution Française, Centre de recherche Espace, sociétés, cultures, Institut universitaire de formation des maîtres (dir.), Maurice Dommanget (1888-1976), citoyen, pédagogue, historien, Beauvais, Archives départementales de l'Oise, 1996.

Annegret Jürgens-Kirchhoff, «Kunst gegen den Krieg im Antikriegsjahr 1924 », dans Jost Dülffer, Gerd Krumeich, Der verlorene Frieden, op. cit., p. 287-310.

Sabine Kienitz, «Beschädigte Helden », dans Jost Dülffer, Gerd Krumeich, Der verlorene Frieden, op. cit., p. 199-214.

Sabine Kienitz, «Körper - Beschädigungen; Kriegsinvalidität und Männlichkeitskonstruktionen in der Weimarer Republik », dans Karen Hagemann, Stefanie Schüler-Springorum, Heimat-Front. Militär und Geschlechterverhältnisse im Zeitalter der Weltkriege, op. cit., p. 188-207.

Katrin Kilian (dir.), Der Erste Weltkrieg in deutschen Bildpostkarten, vol. 66, Berlin, Directmedia Publishing, 2002.

Elke Koch, «,Jeder tut, was er kann fürs Vaterland': Frauen und Männer an der Heilbronner, Heimatfront' », dans Gerhard Hirschfeld, Gerd Krumeich, Dieter Langewiesche, Hans-Peter Ullmann, Kriegserfahrungen..., op. cit., p. 36-52.

Siegfried Kogelfranz, « Schlange vorm Bordell », dans Stephan Burgdorff, Klaus Wiegrefe, Der Erste Weltkrieg. Die Urkatastrophe des 20. Jahrhunderts, op. cit., p. 150-153.

Alan Kramer, «Les 'atrocités allemandes' : mythologie populaire, propagande et manipulations dans l'armée allemande », dans Jean-Jacques Becker, Jay Winter, Gerd Krumeich, Annette Becker, Stéphane AudoinRouzeau, Guerre et cultures (1914-1918), op. cit., p. 147-164.

Gerd Krumeich, «Die Präsenz des Kriegs im Frieden », dans Jost Dülffer, Gerd Krumeich, Der verlorene Frieden, op. cit., p. 7-17.

Gerd Krumeich, « Kriegsfront - Heimatfront », dans Gerhard Hirschfeld, Gerd Krumeich, Dieter Langewiesche, Hans-Peter Ullmann, Kriegserfahrungen..., op. cit., p. 12-19.

Birthe Kundrus, « Geschlechterkriege. Der Erste Weltkrieg und die Deutung der Geschlechterverhältnisse in der Weimarer Republik», dans Karen Hagemann, Stefanie Schüler-Springorum, Heimat-Front. Militär und Geschlechterverhältnisse im Zeitalter der Weltkriege, op. cit., p. 171-187.

Selma Lagerlöf, « Auf uns kommt es jetzt an », dans Gisela Brinker-Gabler, Frauen gegen den Krieg, op. cit., p. 239-241.

Wilhelm Lamszus, Das Menschenschlachthaus. Bilder vom kommenden Krieg, Hamburg, Berlin, Janssen, 1912.

Heinz Lemmermann, Kriegserziehung im Kaiserreich. Studien zur politischen Funktion von Schule und Schulmusik 1890-1918, Lilienthal/Brême, Eres Edition, 1984.

Ulrich Linse, Die anarchistische und anarcho- syndikalistische Jugendbewegung 1918- 1933, Francfort/Main, dipa, 1976.

George Mosse, De la Grande Guerre au totalitarisme: la brutalisation des sociétés européennes, Paris, Hachette-Littératures, 1999.

George Mosse, L'image de l'homme, l'invention de la virilité moderne, Paris, Abbeville, 1997.

Petra Müller, «Sind Frauen friedfertig?», Gegen welchen Krieg - für welchen Frieden? Beiträge zur feministischen Theorie und Praxis, $\mathrm{n}^{\circ} 8,1983$, p. 27-32.

Nicolas Offenstadt, «Contre la guerre. Ce que nous pouvons faire dans nos classes. L'École émancipée et la lutte pour la paix dans l'entre-deux-guerres », dans Institut d'histoire de la Révolution Française, Centre de recherche Espace, sociétés, cultures, Institut universitaire de formation des maîtres, Maurice Dommanget (1888-1976)..., op. cit., p. 79-87. 
Nicolas Offenstadt, «L'image contre la guerre. Autour d'Ernst Friedrich », dans Thérèse Blondet-Bisch, Voir, ne pas voir la guerre, op. cit., p. 271-275.

François Pairault, Images de Poilus. La Grande Guerre en cartes postales, Paris, Tallandier, 2002.

Yannick Ripa, «Armes d'hommes contre femmes désarmées : de la dimension sexuée de la violence dans la guerre civile espagnole », dans Cécile Dauphin, Arlette Farge, De la violence et des femmes, op. cit., p. 131-145.

Anne Roerkohl, « Schlachtfeld Heimat », dans Christine Beil, Der Erste Weltkrieg, op. cit., p. 165-203.

Brunhilde Sauer-Burghard, «'Nur wenige Frauen haben nicht mitgemacht', Feminismus gegen Militarismus?», Gegen welchen Krieg - für welchen Frieden? Beiträge zur feministischen Theorie und Praxis, $\mathrm{n}^{\circ}$ 8, 1983, p. 3345.

Thomas Schirmböck, Roland Scotti, «Gibt es eine pazifistische Kriegsfotographie? », dans Dietrich Harth, Dietrich Schubert, Ronald Michael Schmidt, Pazifismus zwischen den Weltkriegen ..., op. cit., p. 218-228.

Olive Schreiner, «Frauen zahlen die Hauptkosten », dans Gisela Brinker-Gabler, Frauen gegen den Krieg, op. cit., p. 45-50.

Edelgard Spaude-Schulze, Macht das Maul auf! Kinder- und Jugendliteratur gegen den Krieg in der Weimarer Republik, Würzburg, Königshausen \& Neumann, 1990.

Tommy Spree, Ich kenne keine „Feinde“. Der Pazifist Ernst Friedrich; ein Lebensbild, Berlin, Druck- und Verlagsgesellschaft mbH, Druckerei Conrad, 2000.

Françoise Thébaud, « Der Erste Weltkrieg, Triumph der Geschlechtertrennung », dans Georges Duby, Michèle Perrot, Heide Wunder, Geschichte der Frauen, op. cit., p. 33-91.

Bruno Thoß, Hans-Erich Volkmann (dir.), Erster Weltkrieg, Zweiter Weltkrieg. Ein Vergleich. Krieg, Kriegserlebnis, Kriegserfahrung in Deutschland, Paderborn, Schöningh, 2002.

Danièle Voldman, «Les bombardements aériens: une mise à mort du 'guerrier' ? (1914-1945) », dans Cécile Dauphin, Arlette Farge, De la violence et des femmes, op. cit., p. 146-158.

Ignaz Wrobel, « Der bewachte Kriegsschauplatz », Die Weltbühne, n 31, 4.08.1931.

Bérénice Zunino, Ernst Friedrichs Friedenspädagogik mit Kindern und Jugendlichen zwischen 1919 und 1925 , Claire Aslangul, Université Paris IV Sorbonne, 2008. [Mémoire de Master 1]

Bérénice Zunino, Les représentations des femmes et des enfants sur les cartes postales allemandes de la Première Guerre mondiale, Claire Aslangul, Université Paris IV Sorbonne, 2009. [Mémoire de Master 2] 